\section{Is painful knee an independent predictor of mortality in middle-aged women?}

We read with deep interest the article by Kluzek et $a l,{ }^{1}$ which examined whether joint pain or radiographic osteoarthritis (ROA) of the knee and hand is associated with the all-cause and diseasespecific mortality in middle-aged women. The outcome of this prospective community-based Chingford Cohort Study indicated a significantly increased risk of all-cause and cardiovascular disease-specific mortality in women experiencing knee pain with or without ROA, but not in women with ROA alone. It suggests that knee pain, rather than structural changes of osteoarthritis (OA), can be considered as an independent predictor of mortality in middle-aged women. We sincerely appreciate the work performed and the valuable findings obtained by the authors; nevertheless, some issues are worth of further exploration.

First, knee pain was selected as an important evaluative parameter to predict mortality in middle-aged women in this study, but there were several factors that can cause knee pain. A substantial part of knee pain might be related to the knee joint itself and another source of knee pain might result from other musculoskeletal diseases. The most common cause of knee pain is an injury, which can be caused by overuse (bursitis, tendonitis), poor exercise habits (such as not warming up or cooling down or inadequate stretching), a strain, sprain, torn ligament or cartilage, a fracture or a dislocated kneecap. Other problems that can affect the knee include OA (degenerative joint disease) and other types of arthritis; infection or a problem elsewhere in the body (like a pinched nerve), which can cause pain that is felt in the knee. However, the authors had not screened for these conditions. In our opinion, painful knee may be only a clinical manifestation of patients' baseline diseases that can increase mortality drastically. Therefore, it is unclear whether the association between painful knee and increased mortality is attributed to the adverse effects of painful knee or the result of baseline diseases in patients with painful knee. Obviously, further studies addressing these issues are still needed. Furthermore, the cause of painful knee or patients' baseline diseases should also be carefully evaluated. For some, knee pain can be so severe that it limits daily activities. For others, mild knee pain may be a chronic hindrance to the active lifestyle they desire. In either case, chances are that we are dealing with a knee problem that should not be ignored.

Second, this study showed that patients with symptomatic OA were typically of a mature age, and thus more likely to undergo emergency surgery and subject to a greater functional dependency, which implied a higher probability of death. In addition, this study included 821 women for knee analyses with data on both pain and radiographic knee osteoarthritis (KOA). However, according to the sample size calculation, only 64 subjects belonged to the ROA group and 57 patients belonged to the symptomatic OA group. Furthermore, if the women undergoing knee replacement during follow-up $(n=29)$ were excluded, the sample size might become even smaller. The authors explained that "No substantial differences in the results were observed when women who died during the first 12 months of follow-up $(n=2)$, or when women who underwent knee replacement (for knee analysis) during the follow-up $(n=29)$ were excluded". This is understandable. Nevertheless, since the sample size is not big enough, we are not sure whether it is appropriate to draw conclusions with respect to the effect of painful knee.

Third, previous studies have shown that the mortality of middle-aged women were subject to multiple risk factors, including ageing, high frequency of falls, ${ }^{2}$ incident vertebral fractures, ${ }^{3}$ low health-related quality of life, ${ }^{4}$ low socioeconomic status, ${ }^{5}$ employment situation ${ }^{6}$ and high levels of inflammation markers, such as serum C-reactive protein (CRP) levels. ${ }^{7}$ This article did not provide any information on health status, fall history, surgery history except for knee replacement and comorbidities during the follow-up period, which were the most important determinants for mortality. In addition, did the authors have any information on the treatment of pain or KOA during the follow-up period? These data might affect the results, especially with regard to painkillers or other potentially harmful drugs.

Fourth, since all the women were extracted from the same register centre, the four subgroups from hand may be another potential confounding factor for the subgroups from knee, because some women with symptomatic KOA may suffer hand pain or symptomatic hand OA. In addition, as mentioned by the authors, their analysis was likely to underestimate the absolute risk of exposure groups due to the fact that subjects from each group remained in the original group even if they developed pain and/or ROA over time.

Fifth, we respect the results that included and were adjusted for most of the known risk factors affecting mortality. However, we wonder why the authors did not take some other important potential covariates into account, such as other chronic diseases (stroke, chronic obstructive pulmonary disease), educational level (no formal education, primary, secondary, university education), marital status (married, single/separated/widowed) and alcohol consumption (never-drinker, ex-drinker, moderate drinker and excessive drinker), to make the conclusions (independent predictor) more reliable.

Lastly, it should be noted that the median duration of follow-up was 21.7 years (range: 21.2-22.3), but the headline of the paper showed over 23 years of follow-up.

Above all, we respect the great contributions of the authors and we would also be very interested in the authors' response to these issues.

\section{Shu-Guang Gao, ${ }^{1}$ Chao Zeng, ${ }^{1}$ Yi-lin Xiong, ${ }^{1}$ Jie Wei, ${ }^{2,3}$ Guang-Hua Lei ${ }^{1}$}

${ }^{1}$ Department of Orthopaedics, Xiangya Hospital, Central South University, Changsha, Hunan Province, China

${ }^{2}$ Health Management Center, Xiangya Hospital, Central South, University, Changsha, Hunan Province, China

${ }^{3}$ Department of Epidemiology and Health Statistics, School of Public Health, Central South University, Changsha, Hunan Province, China

Correspondence to Professor Guang-Hua Lei, Department of Orthopaedics, Xiangya Hospital, Central South University, Xiangya Road, Changsha, Hunan Province 410008, China; Igh9640@sina.cn

Contributors S-GG: concept, writing; CZ, JW, Y-IX: writing; G-HL: concept, revising. Funding This work was supported by the Natural Science Foundation of China (grant number 81201420, 81272034, 81472130).

Competing interests None declared.

Provenance and peer review Not commissioned; internally peer reviewed.

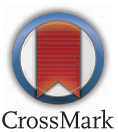

To cite Gao S-G, Zeng C, Xiong Y-lin, et al. Ann Rheum Dis 2016;75:e22.

Accepted 14 December 2015

Published Online First 6 January 2016

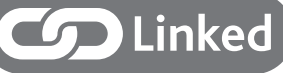

http://dx.doi.org/10.1136/annrheumdis-2015-209029

Ann Rheum Dis 2016;75:e22. doi:10.1136/annrheumdis-2015-209026 


\section{REFERENCES}

1 Kluzek S, Sanchez-Santos MT, Leyland KM, et al. Painful knee but not hand osteoarthritis is an independent predictor of mortality over 23 years follow-up of a population-based cohort of middle-aged women. Ann Rheum Dis 2015. Published Online First 5 Nov 2015. doi:10.1136/annrheumdis-2015-208056

2 Sylliaas H, Idland G, Sandvik L, et al. Does mortality of the aged increase with the number of falls? Results from a nine-year follow-up study. Eur J Epidemiol 2009;24:351-5.

3 Kado DM, Duong T, Stone KL, et al. Incident vertebral fractures and mortality in older women: a prospective study. Osteoporos Int 2003;14:589-94.
4 Otero-Rodríguez A, León-Muñoz LM, Balboa-Castillo T, et al. Change in health-related quality of life as a predictor of mortality in the older adults. Qual Life Res 2010;19:15-23.

5 Kopp MS, Skrabski A, Kawachi I, et al. Low socioeconomic status of the opposite sex is a risk factor for middle aged mortality. J Epidemiol Community Health 2005;59:675-8.

6 Honjo $\mathrm{K}$, Iso $\mathrm{H}$, Ikeda A, et al. Employment situation and risk of death among middle-aged Japanese women. J Epidemiol Community Health 2015;69:1012-17.

7 Ahmadi-Abhari S, Luben RN, Wareham NJ, et al. Seventeen year risk of all-cause and cause-specific mortality associated with C-reactive protein, fibrinogen and leukocyte count in men and women: the EPIC-Norfolk study. Eur J Epidemiol 2013;28:541-50. 